\title{
An Investigation on 3-6-year-old Chinese Children's Perception of "Death"
}

\author{
Yan $\mathrm{Ji}^{1}$, Yanhua $\mathrm{Cao}^{2, *}$, Min $\mathrm{Han}^{3}$ \\ ${ }^{1}$ School of Education, Nantong University, Jiangsu China \\ ${ }^{2}$ School of Foreign Language, Nantong University, Jiangsu China \\ ${ }^{3}$ Maxiang Cuomai Kindergarten Duilong Deqing, Lhasa Tibet, China
}

Copyright $\bigcirc 2017$ by authors, all rights reserved. Authors agree that this article remains permanently open access under the terms of the Creative Commons Attribution License 4.0 International License

\begin{abstract}
Because of the taboo in Chinese culture, there is little research on Chinese children's perception of "death". The research on preschoolers' cognition of "death" could deepen our research on children's cognition process, guide children's life education, and improve the psychologic intervention on the children who experience such torment at their home. This research, with six variables of death concept, applicability, universality, irrevocability, dysfunctionality, causality, and judgment, investigated 180 3-6-year-old Chinese children's general perception of "death", and their cognition of such six sub-concept of "death". The experiment results demonstrated the status of Chinese children's cognition of "death". (1) 3-6-year-old children understand "death" more accurately as they grow older. (2) There is no significant difference in the perception of "death" between different gender children. (3) Children who have experiences with pets have more accurate understanding of "death" than those not. (4) Children have unbalanced understanding of six sub-concept of "death". (5) Children's perception of "death" is self-centered. On the base of such findings, the author advocates to include "death" education into children's daily education, and make use of picture books and the experience with pets to help them understand "death" properly.
\end{abstract}

Keywords "Death" Perception, Life Education, Young Children

\section{Introduction}

"Death", the most distressed topic, is frequently avoided intentionally because it is related to too much sorrow, pain and horror. We often choose to conceal the concept to save children a happy childhood. American early childhood educators found that because of advanced media 3 year olds virtually have come across the word "death". Therefore, "death" is an unavoidable phenomenon to young children. They would keep thinking of and questing the phenomenon they cannot understand. They would ask what "death" is, why people die, and whether they will die.

The research on children's perception of "death" can be traced back to 1930s in western countries. The topic attracted more attention after 1970s [1]. Researchers gave different definition to "death". Most of researchers reached the consensus that "death" is a compound definition which consists of several sub-concepts. Various researchers developed different sub-concepts. Kane (1979) defined, on the base of the analysis on children's perception of "death", nine sub-concept: realization, separation, immobility, irrevocability, causality, dysfunctionality, universality, insensitivity and appearance [2]. Hoffman and Strauss (1985) categorized the concepts into universality, irreversibility, nonfunctionality, causality, and noncorporeal continuation [3]. Speece and Brent (1984) concluded the concepts into three features: universality, irreversibility, and nonfunctionality [4].

Maria Nagy (1948) conducted a classic experiment, and deduced the three stages when children understanding "death", which is in accordance with Piaget's children cognitive development stages. In the first stage (3-5 years old), children think that "death" is temporary, and can be reversed. In the second stage (5-8 years old), children realize that "death" is the end of life, and that it is permanent. In the third stage (9-10 years old), children's cognition of "death" is matured, and they begin to know human is mortal. Nguyen (2002) and Slaughter (2003) found in their researches that preschoolers have a general understanding of "death".

Children's cognitive development occurs in certain culture context. Ross et al claimed that there is an absence of culture background in a great amount of children's biology book [5]. Because of culture difference, Chinese scholars are reluctant to study children's perception of "death". Zhang Xiangkui et al (1998) conducted a research in this area on 3.5-6.5-year children. They tested children's perception of four sub-concepts of "death", irreversibility, unavoidability, 
dysfunctionality and emotionality. The results revealed that at the first stage children could not grasp the meaning of "death". At the second stage, children could explain "death" with certain concrete reasons, which was self-centered and emotional. At the third stage, children could manage to explain "death" with natural reasons, and understand it in a relative abstract term [6].

In Chinese culture, "death" is a taboo, and adults come across difficulty in talking about "death" with kids. Media, in addition, sometimes mislead and even torture children's understanding of "death" with improper description. Therefore, "death" filled children with a mixed feeling of horror, intimidation and even curiosity. Therefore, the investigation into preschoolers' perception of "death" helps with the understanding of children's cognitive development and children's life education. It is critical for effective psychological intervention on children who experience such traumas. This research, on the basis of previous researches, conducted interviews with 180 3-6-year-old children on their perception of six sub-concept of death (applicability, universality, irrevocability, dysfunctionality, causality, and judgment) to explore young children's general perception of "death" and their perception of the six sub-concepts. According to the observation of the researchers in the kindergartens, some young children who raise or raised pets could do better in understanding "death", so experience of pets raising is added as a factor.

\section{Methodology}

\subsection{Participants}

180 3-6-year-old children (from a kindergarten in $\mathrm{N}$ city of Jiangsu Province in China) were recruited as the participants. The participants included 604 years old, 605 years old and 606 years old. The distribution of participants listed in Table 1 and 2.

Table 1. The Distribution of Participants

\begin{tabular}{|c|c|c|c|c|}
\hline Gender & 4 years old & 5years old & 6 years old & Total \\
\hline Male & 30 & 30 & 30 & 90 \\
Female & 30 & 30 & 30 & 90 \\
Total & 60 & 60 & 60 & 180 \\
\hline
\end{tabular}

Table 2. Children's Experience with Pets Raising

\begin{tabular}{|c|c|c|c|c|}
\hline $\begin{array}{c}\text { Pets } \\
\text { Experience }\end{array}$ & 4 years old & 5years old & 6 years old & Total \\
\hline Yes & 26 & 26 & 38 & 90 \\
\hline No & 34 & 34 & 22 & 90 \\
\hline Total & 60 & 60 & 60 & 180 \\
\hline
\end{tabular}

\subsection{Instrument}

The first instrument is picture categorization. 22 pictures of common things, including two of human being (an old man and a kid), five of animals (a fish, a bird, a tortoise, a tiger, and a sleeping cat), five of plants (flowers, grass, trees, mushroom, and cactuses), five of natural objects (river, cobblestones, the sun, the moon and lighting), and five man-made objects (a computer, a lamp, a table and chairs, a plane, and a vehicle), are employed in this instrument. All the 22 pictures need to be categorized into two groups. One is mortal, and the other is of no life.

In order to explore young children's cognition of six sub-concepts of "death", semi-structured interviews were employed to gain the most of the information. The questions in the interview were designed according to the six sub-concepts of "death".

1) The Applicability of Death. Only living things will die. Questions: Will all the things die? What kind of things will die, what kind will not?

2) The Universality of Death. All living things are mortal. Questions: Will all the animals die? (For instance, will cat and dog die?) Will all the plants die? (For example, will all the flowers and grass die?)

3) The Irrevocability of Death. All the dead cannot revive. Questions: Can a dead cat get back to life when she is fed with water and food? Can a dead lamb be revived with magic power?

4) The Dysfunctionality of Death. All the exterior function, like motion and speaking, and interior function, like feeling hungry and sad, will stop working. Questions: Will a cat meow after death? Will a pig want to eat after death?

5) The Causality of Death. The death of a mortal might result from oldness, ailments or exterior factor, like violence. Questions: Will a golden fish die from its disobedience? The gold fish in the picture died. To your understanding, what would be the cause?

6) The Judgment of Death. Tell whether a mortal is dead according to the death definition, like no heart beating or breath. Questions: Could you tell me what is "death"

\subsection{Research Procedure}

Piaget's clinical study was applied in this research. The researcher stepped into classroom and interviewed each child. Before the interview, the researcher obtained general information of children from the teachers of each class, and set the interview time. Through negotiation, the interview time was scheduled at the periods after the breakfast in the morning and after the lunch in the afternoon. The one-on-one interview was conducted in a separate room. The question was semi-constructed, as detailed above, and children's answer was open-ended. The interviewer would add in further questions according to children's response. The whole interview processes were recorded.

First, participating children were required to classify pictures. 22 pictures of common things were used in this process, including two of human being (an old man and a kid), five of animals (a fish, a bird, a tortoise, a tiger, and a 
sleeping cat), five of plants (flowers, grass, trees, mushroom, and cactuses), five of natural objects (river, cobblestones, the sun, the moon and lighting), and five man-made objects (a computer, a lamp, a table and chairs, a plane, and a vehicle).All the 22 pictures were demonstrated randomly before each children, and they were required to categorize the picture into two groups. One is mortal, and the other is of no life. The results were photographed. The researcher added one credit for each correct categorization. There is no score for incorrect one. The maximum score in this part is 22 .

After the first task, participants were interviewed with the semi-structured questions. For each question, mark 0 for incorrect or no response, which indicates the kid did not understand the sub-concept of "death"; mark 1 credit for partial correct response, which indicates the kid could understand the concept to a certain degree; and mark 2 credit for correct response, which indicates the kid had full cognition of the sub-concept.

\section{Results and Analysis}

\subsection{Results}

\subsubsection{Results of Picture Categorization}

Table 3. The Mean of Picture Categorization Credits

\begin{tabular}{|c|c|c|c|c|c|}
\hline Age & Human & Animal & Plants & Natural & Man-made \\
\hline 4 & $0.77(0.86)$ & $1.80(1.97)$ & $1.79(1.69)$ & $3.83(1.78)$ & $3.93(1.84)$ \\
\hline 5 & $1.17(0.75)$ & $2.87(1.61)$ & $1.83(1.88)$ & $4.43(1.17)$ & $4.13(1.53)$ \\
\hline 6 & $1.50(0.68)$ & $3.97(1.45)$ & $3.53(1.80)$ & $4.63(0.67)$ & $4.30(1.15)$ \\
\hline
\end{tabular}

The comparison among groups showed that 4-year olds is different from other two groups significantly $(p<0.05)$ in the categorization of human being and animals, and that 6-year olds is different from other two groups significantly $(p<0.05)$ in the categorization of plants. However, there is no significant difference among the three groups in the categorization of natural and man-made objects. Young children, evidently, influenced by their age in categorizing human being, animal and plant pictures. Even in the classification of natural and man-made objects, where there is no significant difference among three groups, the elder children got higher credits than the younger ones.

\subsubsection{Results of Interview}

(1) 4-6-year olds' general perception of "death"

Table 4. Description of 3-4-year Olds' Perception of "Death"

\begin{tabular}{|c|c|c|c|}
\hline Age & Gender & Mean & $\begin{array}{c}\text { Standard } \\
\text { Deviation }\end{array}$ \\
\hline 4 & Male & 6.67 & 1.80 \\
\hline & Female & 5.80 & 2.65 \\
\hline 5 & Total & 6.23 & 2.27 \\
\hline & Male & 7.47 & 1.81 \\
\hline & Female & 8.20 & 1.37 \\
\hline & Total & 7.83 & 1.62 \\
\hline & Male & 9.87 & 1.51 \\
\hline & Female & 8.93 & 1.94 \\
\hline & Total & 9.40 & 1.77 \\
\hline
\end{tabular}

Figure 1. 4-6-year Olds' Perception of Sub-concepts of "Death" 
Children's perception of "death" and its sub-concepts are displayed in table 4 and figure 1. Statistic comparison demonstrates that there is no significant diffidence between two genders. However, there is significant difference between different age groups $(\mathrm{F}(2,87)=20.659, \mathrm{p}<0.001)$. The results from Scheffe test demonstrate that 6 year old children gained significantly better understanding of "death" $(p<0.001)$, and that 5 year olds are significantly better than 4 year olds $(p<0.001)$. Nevertheless, there is no significant diffidence between two genders. Comparison also reveals that children's cognition of "death" and its sub-concepts develops with their natural growth. Children of all the three ages acquire their understanding of "death" and its sub-concepts alone a certain line: applicability, universality, judgment, irrevocability, causality, and dysfunctionality.

(2) 4-6-year olds' perception of death sub-concepts

Table 5. The Perception Difference between Two Genders of Death Sub-concepts

\begin{tabular}{|c|c|c|c|c|c|}
\hline & Gender & Mean & $\begin{array}{c}\text { Standard } \\
\text { Deviation }\end{array}$ & $\mathrm{F}$ & $\mathrm{P}$ \\
\hline \multirow{3}{*}{ applicability } & Male & 1.00 & 0.71 & \multirow{3}{*}{0.03} & \multirow{3}{*}{0.87} \\
\hline & Female & 0.98 & 0.58 & & \\
\hline & Total & 0.99 & 0.64 & & \\
\hline \multirow{3}{*}{ universality } & Male & 1.11 & 0.57 & \multirow{3}{*}{0.27} & \multirow{3}{*}{0.61} \\
\hline & Female & 1.18 & 0.65 & & \\
\hline & Total & 1.14 & 0.61 & & \\
\hline \multirow{3}{*}{ irrevocability } & Male & 1.42 & 0.58 & \multirow{3}{*}{0.51} & \multirow{3}{*}{0.48} \\
\hline & Female & 1.33 & 0.60 & & \\
\hline & Total & 1.38 & 0.59 & & \\
\hline \multirow{3}{*}{ dysfunctionality } & Male & 1.67 & 0.60 & \multirow{3}{*}{0.25} & \multirow{3}{*}{0.62} \\
\hline & Female & 1.60 & 0.65 & & \\
\hline & Total & 1.63 & 0.63 & & \\
\hline \multirow{3}{*}{ causality } & Male & 1.60 & 0.58 & \multirow{3}{*}{2.00} & \multirow{3}{*}{0.16} \\
\hline & Female & 1.40 & 0.75 & & \\
\hline & Total & 1.50 & 0.67 & & \\
\hline \multirow{3}{*}{ judgment } & Male & \multirow{3}{*}{$\begin{array}{l}1.20 \\
1.16 \\
1.18\end{array}$} & \multirow{3}{*}{$\begin{array}{l}0.63 \\
0.64 \\
0.63\end{array}$} & \multirow{3}{*}{0.11} & \multirow{3}{*}{0.74} \\
\hline & Female & & & & \\
\hline & Total & & & & \\
\hline
\end{tabular}

Table 5 to 7 demonstrated children's perception on the six sub-concepts of death from the perspectives of gender, age and pets experience.

1) Death Applicability: children's perception of death applicability are significantly different between different age groups $(F(2,87)=12.83, p<0.05)$. Children gained more accurate understanding of death applicability as they grow. However, there is no significant difference between two genders, and pets experience also has no significant influence on their perception of this sub-concept.

2) Death Universality. The difference of children's perception of death universality is extremely significant between the three age groups $(\mathrm{F}(2,87)=9.149, \mathrm{p}<0.001)$. Children's understanding of this sub-concept develops as they grow. 6-year olds cognized the sub-concept significantly better than the kids in other two groups. Data also show that girls have better understanding of this sub-concept, but there is no significant difference between the two genders. The group with pets experience also enjoyed a little advantage, but there is also no significant difference.

3) Death Irrevocability. Data show that 6-year olds enjoyed significant advantage over kids in other two groups $(p<0.05)$. Boys got better scores than girls, and children with pets experiences also reported better response than those without, but there is no significant difference.

4) Death Dysfunctionality. Age $(p<0.001)$ and pets experience $(p<0.05)$ had significant influence on children's understanding of "death dysfunctionality". 6-year olds have better understanding than 4-year olds and 5-year olds. Children with pets experience did a better job than those without. However, gender did not have significant affect on children's perception of this sub-concept.

5) Death Causality. Analysis indicates that age affected children's acquisition of death causality significantly $(\mathrm{F}(2,87)=9.405, \quad \mathrm{p}<0.05)$. 4-year olds understand this sub-concept of death significantly better $(p<0.001)$. Children with pets experience scored better than those without, and boys better than girls, but there is no significant difference.

6) Death Judgment. Data did not demonstrate any significant influence of age, gender and pets experience on children's perception of death judgment. However, the elder ones scored better than younger ones, boys better than girls, and those with pets experience better than those without.

Table 6. The Perception Difference between Different Age Groups of Death Sub-concepts

\begin{tabular}{|c|c|c|c|c|c|}
\hline Death Sub-concepts & Age & SD & Mean & $\mathrm{F}$ & $\mathrm{P}$ \\
\hline \multirow{3}{*}{ applicability } & 4 & 0.55 & 0.67 & \multirow{3}{*}{12.83} & \multirow{3}{*}{0.00} \\
\hline & 5 & 0.55 & 0.90 & & \\
\hline & 6 & 0.62 & 1.40 & & \\
\hline \multirow{3}{*}{ universality } & 4 & 0.64 & 0.93 & \multirow{3}{*}{9.15} & \multirow{3}{*}{0.00} \\
\hline & 5 & 0.53 & 1.00 & & \\
\hline & 6 & 0.51 & 1.50 & & \\
\hline \multirow{3}{*}{ irrevocability } & 4 & 0.74 & 1.27 & \multirow{3}{*}{4.56} & \multirow{3}{*}{0.01} \\
\hline & 5 & 0.43 & 1.23 & & \\
\hline & 6 & 0.49 & 1.63 & & \\
\hline \multirow{3}{*}{ dysfunctionality } & 4 & 0.82 & 1.23 & \multirow{3}{*}{12.52} & \multirow{3}{*}{0.00} \\
\hline & 5 & 0.45 & 1.73 & & \\
\hline & 6 & 0.25 & 1.93 & & \\
\hline \multirow{3}{*}{ causality } & 4 & 0.66 & 1.10 & \multirow{3}{*}{9.41} & \multirow{3}{*}{0.00} \\
\hline & 5 & 0.53 & 1.70 & & \\
\hline & 6 & 0.65 & 1.70 & & \\
\hline \multirow{3}{*}{ judgment } & 4 & 0.56 & 1.03 & \multirow{3}{*}{1.22} & \multirow{3}{*}{0.31} \\
\hline & 5 & 0.64 & 1.27 & & \\
\hline & 6 & 0.68 & 1.23 & & \\
\hline
\end{tabular}


Table 7. The Influence of Pets Experience on Children's Perception of Death

\begin{tabular}{|c|c|c|c|c|c|}
\hline & $\begin{array}{c}\text { Pets } \\
\text { Experience }\end{array}$ & Mean & $\begin{array}{c}\text { Standard } \\
\text { Deviation }\end{array}$ & $\mathrm{t}$ & P. \\
\hline \multirow{2}{*}{ applicability } & Yes & 1.02 & 0.62 & \multirow{2}{*}{0.49} & \multirow{2}{*}{0.63} \\
\hline & No & 0.96 & 0.67 & & \\
\hline \multirow{2}{*}{ universality } & Yes & 1.20 & 0.66 & \multirow{2}{*}{0.86} & \multirow{2}{*}{0.39} \\
\hline & No & 1.09 & 0.56 & & \\
\hline \multirow{2}{*}{ irrevocability } & Yes & 1.47 & 0.55 & \multirow{3}{*}{1.43} & \multirow{2}{*}{0.16} \\
\hline & No & 1.29 & 0.63 & & \\
\hline \multirow{2}{*}{ dysfunctionality } & Yes & 1.82 & 0.44 & & \multirow{2}{*}{0.004} \\
\hline & No & 1.44 & 0.72 & \multirow{2}{*}{2.99} & \\
\hline \multirow{2}{*}{ causality } & Yes & 1.58 & 0.66 & & \multirow{2}{*}{0.28} \\
\hline & No & 1.42 & 0.69 & 1.10 & \\
\hline \multirow{2}{*}{ judgment } & Yes & 1.20 & 0.69 & \multirow[t]{2}{*}{0.33} & \multirow[t]{2}{*}{0.74} \\
\hline & No & 1.16 & 0.56 & & \\
\hline
\end{tabular}

\subsection{Analysis and Discussion}

\subsubsection{Analysis on the Results of Picture Categorization}

The results indicate that children could make more precise judgment on "death" as they grew elder, and that they understand the death of animals better than that of plants, which might be the result of children's special feature of cognition and the nature of plants. Children lack of ability to probe into the inner property of things. In the task of human picture categorization, children were required to classify children and elders, and then questioned, "Will you die?" Most children gave negative response, which verified the Egocentrism in Piaget's cognitive theory.

\subsubsection{Children's Perception of "Death"}

4-6 year olds are in the preoperational stage in their cognitive development. Children, at this stage, begin their semiotic thinking, and form primary concepts. Their concept recognition develops as they grow naturally. Therefore, 6-year olds understand "death" much better than the other two age groups.

With the physical and psychological development, children gain better perception of death applicability, universality, irrevocability, dysfunctionality, causality, and judgment. This is in accordance with children's cognitive development process. Children in different age groups displayed cognitive difference, and children of the same age demonstrated fluctuated cognitive level. For instance, young children scored low in their understanding of death applicability, which agrees with the results from previous research. Almost all the 4-year participants thought all the things would die or would not die, which indicates that they could not understand the concept of death applicability. Most 5 -year olds attributed the death to external reasons. For instance, "flowers will die without water," "stone and tortoise will not die for their hard shell," and "the old will die because of their white hair." They still could not understand the nature of death, and their perception of death applicability was still not matured. 6-year olds could generally get the concept of death applicability. They understood the cub-concept, and could tell what kind of things would die according to their texture and life.

Therefore, children are at a critical time in understanding death when they are 5, and it is necessary to facilitate 5-year olds to build up proper concept of death applicability, which can help them develop their love and respect to life, their affection to peers and the society.

\subsubsection{Children's Perception Differences on "Death" between Different Age, Gender and Experience Groups}

The data analysis shows that 4-6-year olds began to understand the "death" concept, which develops as they grow. Children's perception of death is highly related to their age.

Children's cognition of death did not vary significantly between two ages, but boys did get a little bit higher scores than girls. It is interesting that, when asked the reason for the death of a little golden fish, boys would say "it was killed by solders," "it was eaten by a big fish," or "it was killed with a stone." Girls provided answers like, "it was dead because it did not eat," "it was dead since I did not take good care of it," or "it was dead because it left the water." Boys' response displayed "violence" element, while girls' answers reflects their sentimental nature. Hence, gender has some effect on children's understanding of "death concept".

Pets experience is another factor. Data analysis shows that children's pets experience had significant influence on children's mastery of death dysfunctionality concept. Obviously, children can understand that the function of body parts would lost as things die. Children could understand it by analogizing it to their pets' experience. Children's experience is the foundation of their development, and, to a certain extent, children's pets experience is related to children's death concept construction

\section{Conclusion and Implication}

\subsection{Conclusion}

Through data analysis and discussion, following conclusions are reached on 4-6- year old Chinese young children's cognition of death.

1) Children's death cognition develops as they grow. They can gain more and more accurate understanding on the death of living and non-living things.

2) There is no significant difference between children of two genders on their understanding of death. However, boys' incline to use "violence" words in their response, while girls prefer sensitive vocabulary. 
3) Pets experience does not have significant effect on children's perception of death, but children with pets experience score a little bit better than those without.

4) Children acquire the sub-concepts of death in a certain order: death applicability, death universality, death judgment, death irrevocability, death causality, and death dysfunctionality.

5) Children's death perception is also influenced by their egocentrism.

\subsection{Educational Implication}

The features of children's perception of death cast implications for educators' and parents' death and life education on their children.

\section{1) Incorporate Death Education into Children's Life}

Death education has been integrated into elementary education in western countries since the middle of $20^{\text {th }}$ century. With the development of research in this area, American education development put more and more emphasis on death education, and proposed that educates should provide children with proper education material through appropriate teaching methods, which should be suitable to their cognitive development. Death education should also go with health and sanitation education, influencing children in daily education.

4-year olds lack of danger awareness, and educators need to teach children how to protect themselves in daily life. For example, children need to be taught public signs, danger marks, and body protection. 5-year olds began to realize the concept of death. Proper intervention will help them to acquire accurate understanding of death, and soothe death-related emotion. 6-year olds have certain understanding of death, so they need to be guided to learn the relation between life and death.

Hence, in death education, teachers should educate children to

a. learn to protect themselves

b. have proper understanding of death, control their own feelings, and

c. know the relation between death and life.

\section{2) Carry out Death Education with Picture Book}

Talking about and explaining death is always an extremely difficult task for adults. Picture books could provide an effective way for adults to carry out death education. Huang (1994) and Zheng (1999) pointed out that picture books, with its interesting illustrations and plot, are beneficial for young children to let out great pressure, go through depression, and overcome emotional barriers when facing "death" issues.

Educators and parents can choose age appropriate reading materials for sharing, teaching and discussion the concept of death. For instance, Grandpa did not Wear His Suit utilized words and pictures to relate the changing feeling of a boy who lost his grandpa, which can help children to face the death issue, accept the sorrow feeling, and learn to recover over time. A Falling Leaf is another story that explains the balance between death and life with the life cycle of a leaf across four seasons. There are lots of similar picture books that can be employed for death education with children.

3) Enrich Children's Life Experience through Raising Pets

In Guidance for 3-6-Year Olds' Learning and Development, it is advocated that children need to be facilitated to understand the diversity and uniqueness of life, and to learn the life process of birth, growth, production and death through observing, planting and raising activities. Such activities enable children to experience the life cycle, and to develop their awareness of responsibility for life.

Life is the start of education, and life is also the destination of education. Life education is a permanent topic in education, and education keeps pursuing better life. The early childhood stage is a critical period for a human, and it is also the stage to establish the foundation for life-lone education. In this stage, death education is possible and unavoidable. It is necessary to influence life with life, and help children experience life at the beginning of their life, which will inspire their understanding, respect and love for love. This is beneficial for the whole life of the children, and this is the ultimate aim of education.

\section{Acknowledgements}

This research is part of the Educational Research Project of Jiangsu Province, A Research on Parent-child Reading, from a psycho-linguistic perspective.

\section{REFERENCES}

[1] Li, H. (2007). A review on children's cognition of death concept. Research Discussion, 6, 173.

[2] Liu, J. (2010). 6-year olds' death perception and its influential factors. Xi' an: Shangxi Normal University.

[3] Noppe, I. C., \& Noppe, L. D. (2004). Adolescent experiences with death: Letting go of immortality. Journal of Mental Health Counselling, 26, 146-168.

[4] Brent, S. B., Lin, C. H., Speece, M. W., Dong, Q., \& Yang, C. (1996). The Development of the Concept of Death Among Chinese and U.S. Children 3-17 Years of Age: From Binary to "Fuzzy" Concepts?. Omega-journal of Death and Dying, 33(1), 67-83.

[5] Ross, N., Medin, D. L., Coley, J. D., \& Atran, S. (2003). Cultural and experiential differences in the development of folkbiological induction. Cognitive Development, 18(1), 25-47.

[6] Zhang, X. (1998). 3-6- year olds' cognition of death. Psychological Development and Education, 4 (8). 